\title{
Synthesis and antimicrobial studies of some acridinediones and their thiourea derivatives
}

\author{
T. Josephrajan, ${ }^{a}$ V. T. Ramakrishnan, ${ }^{* a}$ G. Kathiravan, ${ }^{b}$ and J. Muthumary ${ }^{b}$ \\ ${ }^{a}$ Department of Organic Chemistry \\ ${ }^{b}$ CAS in Botany \\ University of Madras, Guindy Campus, Chennai- 600 025, India \\ E-mail:vtrk28@yahoo.com
}

\section{Dedicated to Professor S. Swaminathan on his $80^{\text {th }}$ birthday occasion}

(received 31 Aug 04; accepted 14 Dec 04; published on the web 20 Dec 04)

\begin{abstract}
Acridinediones containing thiourea and piperazine moieties, and vanilline derived acridinediones were synthesised. Antimicrobial activities of eight acridinediones were studied against four vibrio isolates.
\end{abstract}

Keywords: Acridinediones, thiourea, geminal coupling, antimicrobial

\section{Introduction}

Anions play numerous fundamental roles in biological and chemical processes 1; for example, the majority of enzymes bind anions as either substrates or cofactors. In addition, the importance of being able to detect and or extract certain environmental anionic pollutants such as nitrate, phosphate and radioactive pertechnetate produced in the nuclear fuel cycle, has only recently been recognized. Recently a chromogenic azophenol- thiourea based anion sensor was reported 2.

Chromogenic receptors for biologically important substrates are one of the current areas of research. A wide variety of chromophores for cations such as alkali and alkaline earth metal ions have been reported. In contrast, only a few chromophores have been reported for the colorimetric determination of anions in the solution. The thiourea group as hydrogen bond donor has recently drawn much interest as a functional group for neutral receptors to recognize mono and dicarboxylate anions, halide ions, sulphates and dihydrogen phosphates 3, 4, 5 .

Molecular recognition 3 is a subject of considerable interest because of its implications in many fields: biology, medicine, environment, etc. In particular, the detection of metal cations involved in biological processes (e.g., sodium, potassium, calcium, magnesium), in clinical 
diagnostics (e.g., lithium, potassium, aluminium), or in pollution (e.g., lead, mercury, cadmium) has received considerable attention. Among the numerous methods employed, fluorescent sensors offer distinct advantages in terms of sensitivity and specificity. 4-Aryl-1, 4dihydropyridines, also known as Hantzsch esters, have proved valuable as drugs for the treatment of cardiovascular disorders 6,7 and constitute an important class of calcium channel blockers 8-11. The relationship between conformation and pharmacological effect in 1,4dihydropyridines (1,4-DHPs) nifedipine-like compounds has been reported 12. Thus, 4-aryl substituted 1,4-DHPs with calcium antagonist properties exist as a boat conformation in which the aryl substituent is in pseudoaxial position, orthogonal to the dihydropyridine plane 13. Previous reports described the synthesis of 1,4-DHPs fused to one 14 or two 15 cyclohexanone rings, which present a positive ionotropic effect promoting (instead of blocking) the entry of calcium to the intracellular space (calcium antagonist effect) 16.

In continuation of our work 17-19 on the synthesis of acridinediones as laser dyes, we herein report the synthesis of acridinediones containing thiourea moiety and piperazine moiety as potential fluorescent chemosensors.

Reaction of tetraketones 1a-c with $p$-nitroaniline in ethanol with a catalytic amount of $\mathrm{P}_{2} \mathrm{O}_{5}$ afforded 10- (4-nitrophenyl)-3,4,6,7,9,10-hexahydro-1,8(2H, 5H) acridinediones (2a-c). The nitro compounds 2a-c were reduced with zinc and $\mathrm{HCl}$ in refluxing ethanol to afford the corresponding 10-(4-aminophenyl)-3,4,6,7,9,10-hexahydro acridinediones 3a-c. The reaction of 10-( $p$-aminophenyl) acridinediones 3a-c with 2-chlorophenyl isothiocyanate in refluxing ethanol did not give the thiourea derivative. Various attempts with other base catalyzed conditions were also unsuccessful. The poor nucleophilicity of the amino group could be due to its position para to the acridine nitrogen in compounds 3a-c. Hence, the tetraketones 1a-c were condensed with 3nitroaniline to afford the 10-(3-nitrophenyl) acridinediones 4a-c which on reduction with $\mathrm{Zn}$ and $\mathrm{HCl}$ afforded the 10-(3-aminophenyl) acridinediones 5a-c. Reaction of compound $5 \mathbf{b}$ with $\mathbf{o}$ chlorophenyl isothiocyanate in refluxing ethanol afforded $\mathrm{N}$-(2-chlorophenyl)-N'-[3-(9-methyl3,4,6,7,9,10-hexahydro-1,8(2H,5H)acridinedione-10-yl)-phenyl] thiourea (6).

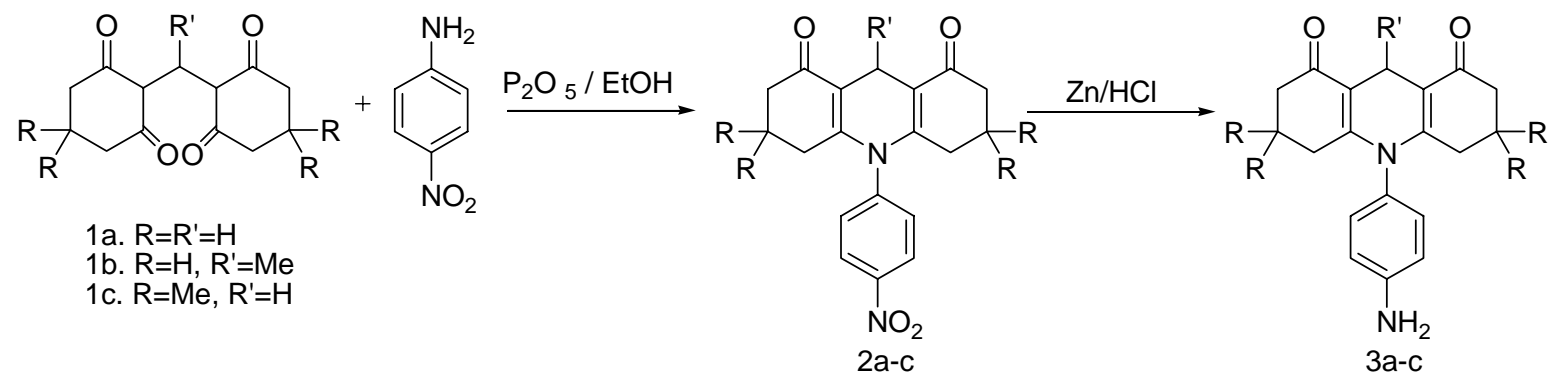

\section{Scheme 1}




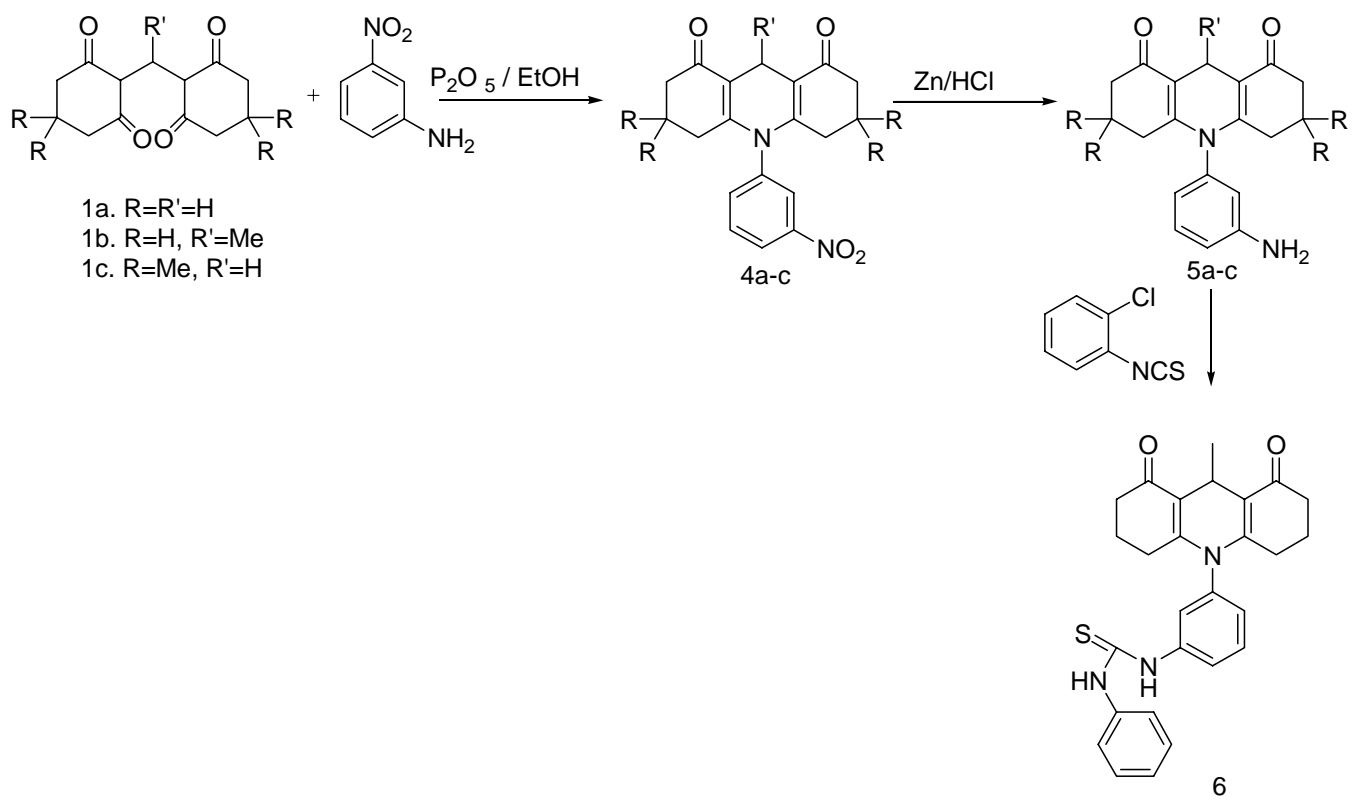

\section{Scheme 2}

Next the reaction of tetraketone 1c with thiosemicarbazide was carried out to afford N(3,3,6,6-tetramethyl-3,4,6,7,9,10-hexahydro-1,8 $(2 \mathrm{H}, 5 \mathrm{H}) \quad$ acridinedione-10-yl)thiourea

(7a). Similarly tetraketone $\mathbf{1 d}$ gave the product $\mathbf{7 b}$. The condensation of $\mathrm{N}$-aminoethyl piperazine with the tetraketones 1e,f in acetic acid was carried out to afford the acridinediones 8a,b in which the $\mathrm{N}$-acetylation of the piperazine ring has also occurred. The two products 8a,b showed good fluorescence. Based on the importance of 4-aryl-dihydropyridines, the tetraketone 1d was reacted with different amines to obtain 9-(4-hydroxy-3-methoxyphenyl acridinediones 9a-f. As examples of hydroxyl group substituted acridinediones, $p$-aminophenol and tyramine were reacted with tetraketones 1a-c to obtain compounds 10a-d.

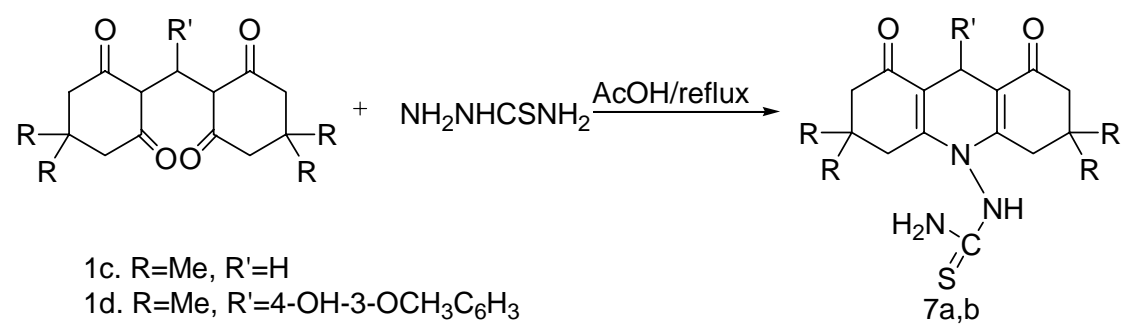

\section{Scheme 3}

Table 1

\begin{tabular}{lll}
\hline Compound & $\mathrm{R}$ & $\mathrm{R}^{\prime}$ \\
\hline $\mathbf{1 c}, \mathbf{7 a}$ & $\mathrm{Me}$ & $\mathrm{H}$ \\
$\mathbf{1 d}, \mathbf{7 b}$ & $\mathrm{Me}$ & $4-\mathrm{OH}-3-\mathrm{OCH}_{3} \mathrm{C}_{6} \mathrm{H}_{3}$ \\
\hline
\end{tabular}




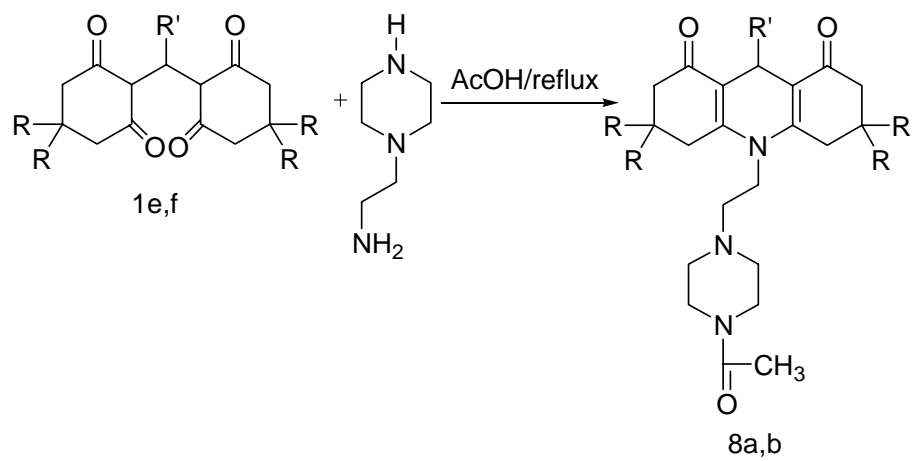

\section{Scheme 4}

Table 2

\begin{tabular}{lll}
\hline Compound & $\mathrm{R}$ & $\mathrm{R}^{\prime}$ \\
\hline 1e, 8a & $\mathrm{Me}$ & $4-\mathrm{CH}_{3} \mathrm{OC}_{6} \mathrm{H}_{4}$ \\
1f, 8b & $\mathrm{Me}$ & $4-\mathrm{ClC}_{6} \mathrm{H}_{4}$ \\
\hline
\end{tabular}

Condensation of dimedone with vanillin furnished 2,2'-(4-hydroxy-3-methoxybenzylidene) bisdimedone 20 (1d), which on reaction with ammonium acetate or amines afforded the respective 9- (4-hydroxy-3-methoxyphenyl)-10-substituted-3, 3, 6, 6-tetramethyl-3, 4, 6, 7, 9, 10hexahydro-1, $8(2 \mathrm{H}, 5 \mathrm{H})$ acridinedione (9a-f).

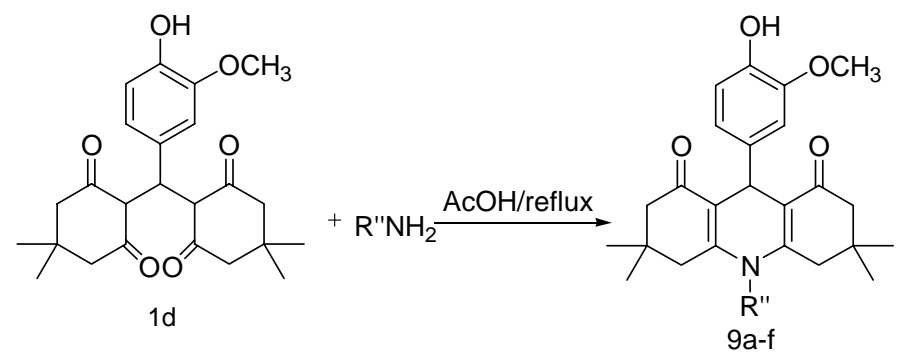

\section{Scheme 5}

\section{Table 3}

\begin{tabular}{ll}
\hline Compound & \\
9 & $\mathrm{R}^{\prime \prime}$ \\
\hline a & $\mathrm{H}$ \\
b & $\mathrm{CH}_{2} \mathrm{COOH}$ \\
c & $4-\mathrm{CH}_{3}-\mathrm{C}_{6} \mathrm{H}_{4}$ \\
d & $4-\mathrm{CH}_{3}-\mathrm{O}-\mathrm{C}_{6} \mathrm{H}_{4}$ \\
e & $\mathrm{Ph}$ \\
f & $3,4-\left(\mathrm{CH}_{3}\right)_{2} \mathrm{C}_{6} \mathrm{H}_{3}$ \\
\hline
\end{tabular}


The NMR spectrum of 9-arylacridinediones 9a-f exhibited geminal coupling of $\mathrm{C}_{2}, \mathrm{C}_{7} \& \mathrm{C}_{4}$, $\mathrm{C}_{5}$ methylene protons; two sets of two doublets were seen for all the compounds 9a-f for the methylene protons. In the IR spectra, all the acridinediones showed absorptions in the $1650 \mathrm{Cm}^{-1}$ region for the carbonyl group due to the vinylogous amide nature.

Reaction of tetraketone 1a,c with $p$-aminophenol afforded the acridinedione 10a,b. Likewise, tetraketones $\mathbf{1 c}, \mathbf{b}$ afforded the acridinediones $\mathbf{1 0}$, $\mathbf{d}$ on reaction with tyramine.

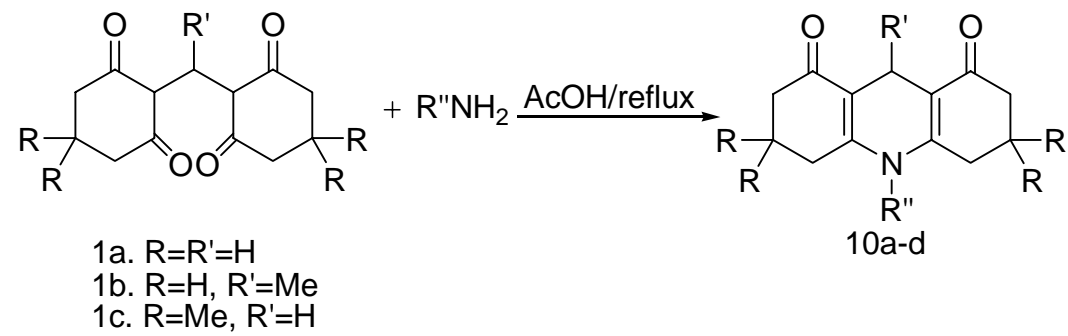

\section{Scheme 6}

Table 4

\begin{tabular}{llll}
$\begin{array}{l}\text { Compound } \\
\mathbf{1 0}\end{array}$ & $\mathrm{R}$ & $\mathrm{R}^{\prime}$ & $\mathrm{R}^{\prime \prime}$ \\
\hline a & $\mathrm{H}$ & $\mathrm{H}$ & $4-(\mathrm{OH}) \mathrm{C}_{6} \mathrm{H}_{4}$ \\
b & $\mathrm{Me}$ & $\mathrm{H}$ & $4-(\mathrm{OH}) \mathrm{C}_{6} \mathrm{H}_{4}$ \\
c & $\mathrm{Me}$ & $\mathrm{H}$ & $4-(\mathrm{OH}) \mathrm{C}_{6} \mathrm{H}_{4} \mathrm{CH}_{2} \mathrm{CH}_{2}$ \\
d & $\mathrm{H}$ & $\mathrm{Me}$ & $4-(\mathrm{OH}) \mathrm{C}_{6} \mathrm{H}_{4} \mathrm{CH}_{2} \mathrm{CH}_{2}$ \\
\hline
\end{tabular}

\section{Biological studies of synthesized acridinediones}

In human pathogenic bacteria, diseases attributed to Vibrio spp. (Vibriosis) 21 are considered to be the most common and significant infectious problems.

Acridines, the earliest known antibiotics, 22-24 are toxic towards bacteria and particularly towards malarial parasite due to their ability to inhibit DNA and RNA synthesis. The eight compounds listed in the tables (3b, 3c, 4a, 9a, 9b, 9d, 10c, 10d) were screened for the antimicrobial activity against different Vibrio isolates under the following conditions.

Method: Well diffusion method, Medium: The nutrient agar medium, Solvent: Chloroform. Concentrations: $50 \mu \mathrm{M}$ and $100 \mu \mathrm{M}$. Condition: 24 hours at $24-28^{\circ} \mathrm{C}$, Standard: The antibiotic Streptomycin

The nutrient agar medium, $20 \mathrm{~mL}$ was poured into the sterile petri dishes. To the solidified plates, wells were made using a sterile cork borer $10 \mathrm{~mm}$ in diameter. The 24 hour subcultured bacteria was inoculated in the petri-plates, with a sterile cotton swab dipped in the nutrient broth medium. After inoculating, the compounds were dissolved separately with the chloroform solvent and poured into the wells with varying concentrations ranging from $50 \& 100 \mu \mathrm{M}$ using a micropipette. 
The plates were left over for 24 hours at $24-28{ }^{\circ} \mathrm{C}$. The antibiotic Streptomycin was used as a standard for comparative study. The percentage of inhibition was calculated by the formula

$$
\% \text { Inhibition }=\quad \frac{\text { Diameter of the inhibition zone } \mathrm{x} 100}{\text { Diameter of the petri-plate }}
$$

From this data, it has been found that all the compounds tested showed broad spectrum of inhibitory properties. The compound 9b \& 9d showed good inhibition zone on the pathogen Vibrio isolate-I and moderate activity against the other isolates. The rest of the compounds have moderate activity against Vibrio isolate-I. The activity of compounds were diminished with Vibrio isolate-IV. Compounds 9a \& 10c showed good activity against Vibrio isolate-II.

Table 5. Effect of acridinediones $(50,100 \mathrm{uM})$ concentrations on Vibrio isolates I-IV

\begin{tabular}{llllllllll}
\hline $\begin{array}{l}\text { Vibrio } \\
\text { Isolate }\end{array}$ & $\begin{array}{l}\text { Standard } \\
\mathrm{A} \mathrm{uM} \\
(\% \mathrm{I})\end{array}$ & $\mathbf{3 b}$ & $\mathbf{3 c}$ & $\mathbf{4 a}$ & $\mathbf{9 a}$ & $\mathbf{9 b}$ & $\mathbf{9 d}$ & $\mathbf{1 0 c}$ & $\mathbf{1 0 d}$ \\
\hline I & & 55 & 41 & 58 & 53 & 66 & 70 & 49 & 48 \\
& 27.5 & $(64$ & 54 & 63 & 62 & 71 & 81 & 58 & $60)$ \\
II & & 50 & 47 & 69 & 72 & 63 & 53 & 84 & 55 \\
& 23.1 & $(62$ & 54 & 77 & 80 & 70 & 62 & 90 & $67)$ \\
III & & 39 & 44 & 39 & 38 & 48 & 42 & 53 & 38 \\
& 19.3 & $(47$ & 65 & 50 & 52 & 54 & 55 & 59 & $46)$ \\
IV & & 38 & 55 & 36 & 44 & 31 & 29 & 33 & 39 \\
& 21.8 & $(49$ & 68 & 52 & 60 & 46 & 38 & 44 & $52)$ \\
\hline
\end{tabular}

A: Antibiotic streptomycin; I: Inhibition zone; \%I: Percentage of Inhibition

\section{Experimental Section}

General Procedures. Melting points were determined by using a Toshniwal melting point apparatus in an open capillary tube and are uncorrected. IR spectra were recorded in Nicolet Impact 400 FT-IR spectrophotometer. ${ }^{1} \mathrm{H}$ NMR and ${ }^{13} \mathrm{C}$ NMR were recorded on Jeol GSX $400 \mathrm{MHz}$ using TMS as internal standard. GC/MS data were obtained from a Jeol-DX-303 spectrometer. Microanalyses were performed in a Perkin-Elmer 240B element analyzer.

Preparation of 4- hydroxy-3-methoxybenzylidene bisdimedone (1d). To a solution of dimedone ( $4.0 \mathrm{~g}, 0.029 \mathrm{~mol})$ in aq. methanol was added vanillin $(2.17 \mathrm{~g}, 0.014 \mathrm{~mol})$ and warmed until the solution became cloudy. The 4-hydroxy-3-methoxybenzylidene bisdimedone (1d) started to separate out. The reaction mixture was diluted with water $(50 \mathrm{ml})$ and allowed to 
stand overnight; the tetraketone 1d was collected by filtration and dried and recrystallized from methanol.

Yield: 5.56 g, 94 \%; mp; $181-183^{\circ} \mathrm{C}$ (Lit. mp. 195.5-196.5 C) [20]

All other tetraketones were prepared as per literature procedure. [20]

\section{General procedure for the synthesis of nitroacridines $\mathbf{2 b , c} \& \mathbf{4 b , c}$}

A mixture of tetraketone $1(\mathrm{a}-\mathrm{c})(3.4 \mathrm{mmol})$ and nitroaniline $(3.4 \mathrm{mmol})$ in ethanol $(40 \mathrm{ml})$ was stirred at room temperature with a catalytic amount of $\mathrm{P}_{2} \mathrm{O}_{5}$ for 10 hours. The reaction mixture was concentrated and poured into cold water $(100 \mathrm{ml})$. The yellow solid obtained was filtered, dried and recrystallized from chloroform-methanol to obtain the respective acridinedione. Syntheses of compounds $2 \mathrm{a}$ [17] and 4a [19] were reported earlier.

9-Methyl-10- (4-nitrophenyl)-3,4,6,7,9,10-hexahydro-1,8(2H, 5H)acridinedione (2b). Yield $69 \%$; yellow; mp 210-212 ${ }^{\circ} \mathrm{C}$; IR $(\mathrm{KBr}) 1637,1580,1531,1374,1345 \mathrm{~cm}^{-1} ;{ }^{1} \mathrm{H}$ NMR $(90$ $\left.\mathrm{MHz} \mathrm{CDCl}_{3}\right) \delta 1.01(\mathrm{~d}, 3 \mathrm{H}, J=6.1 \mathrm{~Hz}), 1.80-2.06\left(\mathrm{~m}, 8 \mathrm{H}, \mathrm{C}_{2}, \mathrm{C}_{3}, \mathrm{C}_{6} \& \mathrm{C}_{7}-\mathrm{CH}_{2}\right), 2.25-2.40(\mathrm{~m}$, $\left.4 \mathrm{H}, \mathrm{C}_{4} \& \mathrm{C}_{5}-\mathrm{CH}_{2}\right), 4.13$ (q, 1H, C $\left.\mathrm{C}_{9} \mathrm{CH}\right), 7.51$ (d, 2H, J=9.5 Hz, Ar-H), 8.38 (d, 2H, J = 9.5 Hz, Ar-H); MS (\%) $352\left(\mathrm{M}^{+}, 10.1\right), 338$ (83.3), 337 (100),336 (3.1), 335 (3.6), 321 (4.7), 309 (3.2), 307 (20.4), 291(89.7), 55 (48). $\mathrm{C}_{20} \mathrm{H}_{20} \mathrm{~N}_{2} \mathrm{O}_{4}$ requires: C, 68.16; H, 5.72; N, 7.94; found: C, 68.07; H., 5.46; N, 7.68 .

10-(4-Nitrophenyl)-3,3,6,6-tetramethyl-3,4,6,7,9,10-hexahydro-1,8(2H,5H)acridinedi one (2c). Yield $85 \%$; yellow; mp 265-267 ${ }^{\circ} \mathrm{C}$; IR (KBr) 1660, 1580, 1530, 1375, $1350 \mathrm{~cm}^{-1} ;{ }^{1} \mathrm{H}$ NMR $\left(90 \mathrm{MHz}, \mathrm{CDCl}_{3}\right) \delta 0.97$ (s, 12H, gem-dimethyl), $1.80\left(\mathrm{~s}, 4 \mathrm{H}, \mathrm{C}_{2} \& \mathrm{C}_{7}-\mathrm{CH}_{2}\right), 2.25\left(\mathrm{~s}, 4 \mathrm{H}, \mathrm{C}_{4}\right.$ \& $\left.\mathrm{C}_{5}-\mathrm{CH}_{2}\right), 3.21\left(\mathrm{~s}, 2 \mathrm{H}, \mathrm{C}_{9}-\mathrm{CH}_{2}\right), 7.52(\mathrm{~d}, 2 \mathrm{H}, \mathrm{J}=9.8 \mathrm{~Hz}, \mathrm{Ar}-\mathrm{H}), 8.41$ (d, 2H, J=9.8 Hz, Ar-H); MS (\%) $394\left(\mathrm{M}^{+}, 38.2\right), 393$ (8.2), 377(9.6), 364(10.3), 257(13.8), 256(8.7), 138 (13.2), 105 (100), 83 (26.8), 69 (40). $\mathrm{C}_{23} \mathrm{H}_{26} \mathrm{~N}_{2} \mathrm{O}_{4}$ requires: $\mathrm{C}, 70.03 ; \mathrm{H}, 6.64 ; \mathrm{N}, 7.10$; found: $\mathrm{C}, 69.76 ; \mathrm{H}, 6.47$; $\mathrm{N}$, 6.93.

9-Methyl-10- (3-nitrophenyl)-3,4,6,7,9,10-hexahydro-1,8(2H, $5 H$ ) acridinedione (4b). Yield 79 \%; yellow; mp $197-199{ }^{\circ} \mathrm{C}$; IR (KBr) 1665, 1575, 1535, 1368, $1340 \mathrm{~cm}^{-1}$; ${ }^{1} \mathrm{H}$ NMR (90 MHz, $\left.\mathrm{CDCl}_{3}\right) \delta 1.06\left(\mathrm{~d}, 3 \mathrm{H}, J=7.35 \mathrm{~Hz}, \mathrm{CH}_{3}\right), 1.9-2.06\left(\mathrm{~m}, 8 \mathrm{H}, \mathrm{C}_{2}, \mathrm{C}_{3}, \mathrm{C}_{6} \& \mathrm{C}_{7}-\mathrm{CH}_{2}\right), 2.30-2.51(\mathrm{~m}$, $\left.4 \mathrm{H}, \mathrm{C}_{4} \& \mathrm{C}_{5}-\mathrm{CH}_{2}\right), 4.11\left(\mathrm{q}, 1 \mathrm{H}, \mathrm{C}_{9}-\mathrm{CH}\right), 7.51-8.53$ (m, 4H, Ar-H); MS (\%) $352\left(\mathrm{M}^{+}, 4.1\right), 350$ (4.3), 337 (77.6), 336 (7.4), 335 (27.8), 321 (3.3), 307 (10.8), 291 (24), 217 (100), 215 (25.6), 189 (5.6), 175 (7.5), 161 (4.5), 159 (3.7), 145 (4.8), 138 (9.4), 105 (5.2), 55 (23.9). $\mathrm{C}_{20} \mathrm{H}_{20} \mathrm{~N}_{2} \mathrm{O}_{4}$ requires: $\mathrm{C}, 68.16 ; \mathrm{H}, 5.72 ; \mathrm{N}, 7.94$; found: $\mathrm{C}, 68.02 ; \mathrm{H}, 5.84 ; \mathrm{N}, 7.78$.

10-(3-Nitrophenyl)-3,3,6,6-tetramethyl--3,4,6,7,9,10-hexahydro-1,8(2H,5H)acridine dione (4c). Yield $78 \%$; yellow; mp above $300{ }^{\circ} \mathrm{C}$; IR (KBr) 1632, 1545, 1534, 1387, $1365 \mathrm{~cm}^{-1}$; ${ }^{1} \mathrm{H}$ NMR $\left(90 \mathrm{MHz}, \mathrm{CDCl}_{3}\right) \delta 0.94$ (s, 12H, gem-dimethyl), 1.75 (br s, 4H, $\mathrm{C}_{2} \& \mathrm{C}_{7}-\mathrm{CH}_{2}$ ), 2.10-2.40 (2d, $\left.4 \mathrm{H}, \mathrm{C}_{4} \& \mathrm{C}_{5}-\mathrm{CH}_{2}\right), 3.25$ (s, 2H, $\left.\mathrm{C}_{9}-\mathrm{CH}_{2}\right), 7.66-8.41(\mathrm{~m}, 4 \mathrm{H}, \mathrm{Ar}-\mathrm{H})$; MS (\%) $394\left(\mathrm{M}^{+}, 100\right), 393$ (23.4), 379 (18), 378 (11.5), 377 (36.2), 365 (7.9), 364 (14.1), 363 (9.2), 351(6.9), 349 (16), 348 (12.8), 347 (26.8), 337 (9.7), 331 (12.4), 323 (9.7), 256 (8.8), 83 (21.6), 69 (10.2), 55 (34.4). $\mathrm{C}_{23} \mathrm{H}_{26} \mathrm{~N}_{2} \mathrm{O}_{4}$ requires: $\mathrm{C}, 70.03 ; \mathrm{H}, 6.64 ; \mathrm{N}, 7.10$; found: $\mathrm{C}, 69.80 ; \mathrm{H}, 6.85 ; \mathrm{N}, 7.32$. 


\section{General procedure for the synthesis of aminoacridinediones3a-c \& 5a-c}

The nitroacridinedione 2a $(1.0 \mathrm{~g}, 2.95 \mathrm{mmol})$ was dissolved in ethanol $(40 \mathrm{ml})$ and $5 \mathrm{~g}$ of zinc dust was added; a few drops of con. $\mathrm{HCl}$ was added and.the mixture refluxed for 5 hours. After the completion of the reaction, the zinc dust was filtered off, the filtrate concentrated, and water $(50 \mathrm{ml})$ added. The solid was filtered, dried, and purified by column chromatography using neutral alumina and eluting with chloroform to isolate the amino compound 3a.

10-(4-Aminophenyl)-3,4,6,7,9,10-hexahydro-1,8(2H, 5H) acridinedione (3a). Yield 71 \%; brown; mp 251-253 ${ }^{\circ} \mathrm{C}$; IR (KBr) 3433, 3351, 1631, 1514, $1384 \mathrm{~cm}^{-1}$; ${ }^{1} \mathrm{H}$ NMR (90 MHz, $\left.\mathrm{CDCl}_{3}\right) \delta 1.80-2.10\left(\mathrm{~m}, 8 \mathrm{H}, \mathrm{C}_{2}, \mathrm{C}_{3}, \mathrm{C}_{6} \& \mathrm{C}_{7}\right), 2.20-2.40\left(\mathrm{~m}, 4 \mathrm{H}, \mathrm{C}_{4}, \mathrm{C}_{5}\right), 3.22\left(\mathrm{~s}, 2 \mathrm{H}, \mathrm{C}_{9}-\mathrm{CH}_{2}\right)$, 4.01 (br s, $2 \mathrm{H}, \mathrm{NH}_{2}$, exchanged with $\mathrm{D}_{2} \mathrm{O}$ ), 6.73-7.03 (2d, 4H, Ar-H); MS (\%) 308( $\left.\mathrm{M}^{+}, 100\right), 307$ (100), 306 (46), 292 (4.8), 291 (13.5), 279 (7.3), 277(8.4), 265 (6), 251 (11), 249 (8.8), 223 (5.2), 214 (4), 202 (7.4), 195 (10.6), 154 (3), 106 (49.8). $\mathrm{C}_{19} \mathrm{H}_{20} \mathrm{~N}_{2} \mathrm{O}_{2}$ requires: C, 74.00; H, 6.53; N, 9.08; found: C, 74.43; H, 6.86; N, 8.80.

10-(4-Aminophenyl)-9-methyl-3,4,6,7,9,10-hexahydro-1,8(2H,5H)acridinedione (3b). Yield $77 \%$; greenish yellow; mp 260-262 ${ }^{\circ} \mathrm{C}$; IR (KBr) 3420, 3340, 1680, 1560, $1380 \mathrm{~cm}^{-1}$; ${ }^{1} \mathrm{H} \mathrm{NMR}$ $\left(400 \mathrm{MHz}, \mathrm{CDCl}_{3}\right) \delta 0.99\left(\mathrm{~d}, 3 \mathrm{H}, J=6.3 \mathrm{~Hz}, \mathrm{CH}_{3}\right), 1.75-2.44\left(\mathrm{~m}, 12 \mathrm{H}, \mathrm{C}_{2}, \mathrm{C}_{3}, \mathrm{C}_{4}, \mathrm{C}_{5}, \mathrm{C}_{6} \& \mathrm{C}_{7^{-}}\right.$ $\left.\mathrm{CH}_{2}\right), 4.12-4.17$ (q, 1H, $\left.J=6.26 \mathrm{~Hz}, \mathrm{C}_{9}-\mathrm{CH}\right), 6.74(\mathrm{~d}, 2 \mathrm{H}, J=7.8 \mathrm{~Hz}, \mathrm{Ar}-\mathrm{H}), 7.10(\mathrm{~d}, 2 \mathrm{H}, J=$ $\mathrm{Hz}, \mathrm{Ar}-\mathrm{H}) ;{ }^{13} \mathrm{C}$ NMR $\left(100.4 \mathrm{MHz}, \mathrm{CDCl}_{3}\right) \delta 21.3\left(\mathrm{CH}_{2}\right), 21.5(\mathrm{CH}), 22.8,\left(\mathrm{CH}_{3}\right), 28.0\left(\mathrm{CH}_{2}\right)$, $36.8\left(\mathrm{CH}_{2}\right), 115.5(\mathrm{CH}), 116.7(\mathrm{C}), 129.2(\mathrm{C}), 129.9(\mathrm{C}), 130.4(\mathrm{CH}), 147.1(\mathrm{C}), 196.6(\mathrm{C})$; .MS (\%) $307\left(\mathrm{M}^{+}-\mathrm{CH}_{3}, 100\right) . \mathrm{C}_{20} \mathrm{H}_{22} \mathrm{~N}_{2} \mathrm{O}_{2}$ requires: $\mathrm{C}$, 74.50; H, 6.87; N 8.68; found: C, 74.09; $\mathrm{H}$, $6.73 ; \mathrm{N}, 8.47$.

10-(4-Aminophenyl)-3,3,6,6-tetramethyl-3,4,6,7,9,10-hexahydro-1,8(2H,5H) acridine dione (3c). Yield $76 \%$; brown; mp 290-292 ${ }^{\circ}$; IR (KBr) 3432, 3320, 1631, 1555, $1385 \mathrm{~cm}^{-1}$; ${ }^{1} \mathrm{H}$ NMR $\left(400 \mathrm{MHz}, \mathrm{CDCl}_{3}\right) \delta 0.94$ (s, 12H, gem-dimethyl), 1.85 (s, 4H, $\left.\mathrm{C}_{2}, \mathrm{C}_{7}-\mathrm{CH}_{2}\right), 2.21\left(\mathrm{~s}, 4 \mathrm{H}, \mathrm{C}_{4}, \mathrm{C}_{5^{-}}\right.$ $\left.\mathrm{CH}_{2}\right), 3.21\left(\mathrm{~s}, 2 \mathrm{H}, \mathrm{C}_{9}-\mathrm{CH}_{2}\right), 4.03\left(\mathrm{~s}, 2 \mathrm{H}, \mathrm{NH}_{2}\right), 6.74(\mathrm{~d}, 2 \mathrm{H}, J=8.3 \mathrm{~Hz}, \mathrm{Ar}-\mathrm{H}), 6.89$ (d, 2H, J = $8.3 \mathrm{~Hz}, \mathrm{Ar}-\mathrm{H}) ;{ }^{13} \mathrm{C} \mathrm{NMR}\left(100.4 \mathrm{MHz}, \mathrm{CDCl}_{3}\right) \delta 18.5\left(\mathrm{C}_{9}-\mathrm{CH}_{2}\right), 28.3\left(\mathrm{CH}_{3}\right), 32.1(\mathrm{C}), 41.7\left(\mathrm{C}_{2^{-}}\right.$ $\left.\mathrm{CH}_{2}\right), 49.9\left(\mathrm{C}_{4}-\mathrm{CH}_{2}\right), 110.6(\mathrm{C}), 115.5(\mathrm{CH}), 129.4(\mathrm{C}), 130.4(\mathrm{CH}), 146.9(\mathrm{C}), 151.9(\mathrm{C}), 196.8$ (C); MS (\%) 364(M+ $\left.{ }^{+}, 98.2\right), 363$ (33.8), 349(17.4), 348 (14.7), 347 (29.8), 333 (9.8), 320 (10.8), 280 (10.1), 195 (15.1), 106 (9.3). $\mathrm{C}_{23} \mathrm{H}_{28} \mathrm{~N}_{2} \mathrm{O}_{2}$ requires $\mathrm{C}, 75.79 ; \mathrm{H}, 7.74 ; \mathrm{N}, 7.68$; found $\mathrm{C}$, 75.44; H, 7.69; N, 7.49.

10-(3-Aminophenyl)-3,4,6,7,9,10-hexahydro-1,8(2H,5H) acridinedione (5a). Yield $71 \%$; brown; mp 247-249 ${ }^{\circ} \mathrm{C}$; IR (KBr) 3435, 3350, 1665, 1575, $1360 \mathrm{~cm}^{-1}$; ${ }^{1} \mathrm{H}$ NMR (400 MHz, $\left.\mathrm{CDCl}_{3}\right) \delta 0.94\left(\mathrm{~s}, 12 \mathrm{H}\right.$, gem-dimethyl), 1.82-2.56 (m, 12H, $\left.\mathrm{C}_{2}, \mathrm{C}_{3}, \mathrm{C}_{4}, \mathrm{C}_{5}, \mathrm{C}_{6} \& \mathrm{C}_{7}-\mathrm{CH}_{2}\right), 3.21$ (s, $2 \mathrm{H}, \mathrm{C}_{9}-\mathrm{CH}_{2}$ ), 3.98 (br s, 2H, Ar- $\mathrm{NH}_{2}$ ), 6.45-7.27 (m, 4H, Ar-H); MS (\%) 308 (M+1, 80), 307 (28), 292 (8.4), 251 (20), 214 (10), 195 (15), 154 (7), 106 (65). $\mathrm{C}_{19} \mathrm{H}_{20} \mathrm{~N}_{2} \mathrm{O}_{2}$ requires: C, 74.00; $\mathrm{H}$, $6.53 ; \mathrm{N}, 9.08$; found $\mathrm{C}, 73.72 ; \mathrm{H}, 6.68 ; \mathrm{N}, 8.87$.

10-(3-Aminophenyl)-9-methyl-3,4,6,7,9,10-hexahydro-1,8(2H,5H)acridinedione (5b). Yield $75 \%$; brown; mp 274-276 ${ }^{\circ} \mathrm{C}$; IR (KBr) 3420, 3378, 1675, 1550, $1360 \mathrm{~cm}^{-1}{ }^{1} \mathrm{H}$ NMR (400 MHz, $\left.\mathrm{CDCl}_{3}-\mathrm{DMSO}-d_{6}\right): \delta 0.91\left(\mathrm{~d}, 3 \mathrm{H}, J=6.35 \mathrm{~Hz}, \mathrm{CH}_{3}\right), 1.78-1.91\left(\mathrm{~m}, 4 \mathrm{H}, \mathrm{C}_{3}, \mathrm{C}_{6}-\mathrm{CH}_{2}\right), 2.06-2.35$ $\left(\mathrm{m}, 8 \mathrm{H}, \mathrm{C}_{2}, \mathrm{C}_{4}, \mathrm{C}_{5} \& \mathrm{C}_{7}-\mathrm{CH}_{2}\right), 3.99$ (q, $\left.1 \mathrm{H}, J=6.8 \mathrm{~Hz}, \mathrm{C}_{9}-\mathrm{CH}\right), 4.96$ (br s, 2H, NH ), 6.43-7.89 
(m, $4 \mathrm{H}, \mathrm{Ar}-\mathrm{H}) . \mathrm{C}_{20} \mathrm{H}_{22} \mathrm{~N}_{2} \mathrm{O}_{2}$ requires: C, 74.50; H, 6.87; N, 8.68; found $\mathrm{C}, 74.37 ; \mathrm{H}, 6.92 ; \mathrm{N}$, 8.54 .

10-(3-Aminophenyl)-3,3,6,6-tetramethyl-3,4,6,7,9,10-hexahydro-1,8(2H,5H) acridine dione (5c).Yield $71 \%$; brown; mp 252-254 ${ }^{\circ} \mathrm{C}$; IR (KBr) 3432, 3351, 2949, 1631, $1385 \mathrm{~cm}^{-1}$; ${ }^{1} \mathrm{H}$ NMR $\left(400 \mathrm{MHz}, \mathrm{CDCl}_{3}\right) \delta 0.94\left(\mathrm{~s}, 12 \mathrm{H}\right.$, gemdimethyl), $1.90\left(\mathrm{~s}, 4 \mathrm{H}, \mathrm{C}_{2} \& \mathrm{C}_{7}-\mathrm{CH}_{2}\right), 2.21\left(\mathrm{~s}, 4 \mathrm{H}, \mathrm{C}_{4}\right.$ \& $\left.\mathrm{C}_{5}-\mathrm{CH}_{2}\right), 3.21\left(\mathrm{~s}, 2 \mathrm{H}, \mathrm{C}_{9}-\mathrm{CH}_{2}\right), 3.98\left(\mathrm{~s}, 2 \mathrm{H}, \mathrm{Ar}_{-} \mathrm{NH}_{2}\right) ; 6.45-7.27$ (m, 4H, Ar-H); ${ }^{13} \mathrm{C}$ NMR (100.4 $\left.\mathrm{MHz}, \mathrm{CDCl}_{3}\right): \delta 18.5\left(\mathrm{C}_{9}-\mathrm{CH}_{2}\right), 28.3\left(\mathrm{CH}_{3}\right), 32.3(\mathrm{C}), 41.5\left(\mathrm{C}_{2}-\mathrm{CH}_{2}\right), 50.0\left(\mathrm{C}_{4}-\mathrm{CH}_{2}\right), 110.6(\mathrm{C})$, $115.5(\mathrm{CH}), 115.7(\mathrm{CH}), 119.4(\mathrm{CH}), 130.5(\mathrm{CH}), 140.1(\mathrm{C}), 148.0(\mathrm{C}), 151.2(\mathrm{C}), 196.7(\mathrm{C})$; MS (\%) $364\left(\mathrm{M}^{+}, 100\right), 363$ (14), 349 (17.1), 348 (13), 280 (6), 134 (35.9). $\mathrm{C}_{23} \mathrm{H}_{28} \mathrm{~N}_{2} \mathrm{O}_{2}$ requires: C, 75.79; H, 7.74; N, 7.68; found: C, 75.47; H, 7.83; N, 7.52.

$\mathrm{N}$-(2-Chlorophenyl)-N'-[3-(9-methyl-3,4,6,7,9,10-hexahydro-1,8(2H,5H)acridinedi one-10yl)-phenyl] thiourea (6). The acridinedione $5 \mathrm{~b}(1.0 \mathrm{~g}, 3.1 \mathrm{mmol})$ and 2-chlorophenyl isothiocyanate $(0.52 \mathrm{~g}, 3.1 \mathrm{mmol})$ on refluxing in ethanol for 9 hours furnished the thiourea 6.Yield $72 \%$; brown; mp 238-240 ${ }^{\circ} \mathrm{C}$; IR (KBr) 3425, 3378, 1665, 1441, $1372 \mathrm{~cm}^{-1} .{ }^{1} \mathrm{H} \mathrm{NMR}$ $\left(400 \mathrm{MHz}, \mathrm{CDCl}_{3}\right) \delta 1.01\left(\mathrm{~d}, 3 \mathrm{H}, \mathrm{J}=7.8 \mathrm{~Hz}, \mathrm{CH}_{3}\right), 1.81-2.43\left(\mathrm{~m}, 12 \mathrm{H}, \mathrm{C}_{2}, \mathrm{C}_{3}, \mathrm{C}_{4}, \mathrm{C}_{5}, \mathrm{C}_{6} \& \mathrm{C}_{7^{-}}\right.$ $\mathrm{CH}_{2}$ ), 3.48 (br s, 2H, NH, exchanged with $\left.\mathrm{D}_{2} \mathrm{O}\right), 4.12\left(\mathrm{q}, 1 \mathrm{H}, \mathrm{J}=7.6 \mathrm{~Hz}, \mathrm{C}_{9}-\mathrm{CH}\right), 6.51-7.81$ (m, 8H, Ar-H); MS (\%) 380 (7.1), 379 (27.5) , 322 (5.5), 307 (100), 216 (3.4), 214 (4.7). $\mathrm{C}_{27} \mathrm{H}_{26} \mathrm{~N}_{3} \mathrm{O}_{2} \mathrm{SCl}$ requires: $\mathrm{C}, 65.90 ; \mathrm{H}, 5.32 ; \mathrm{N}, 8.53$; found: $\mathrm{C}, 65.59 ; \mathrm{H}, 5.30 ; \mathrm{N}, 8.32$.

\section{General procedure for the synthesis of thioureas 7a,b}

A mixture of the tetraketone 1c (1d) $(3.4 \mathrm{mmol})$ and thiosemicarbazide $(3.4 \mathrm{mmol})$ was refluxed in acetic acid $(15 \mathrm{ml})$ for 14 hours. The reaction mixture was cooled and poured into crushed ice. The solid obtained was $\mathrm{CHCl}_{3}-\mathrm{MeOH}(8: 2)$, to isolate the thiourea 7a (7b).

$N$-(3,3,6,6-Tetramethyl-3 filtered and purified by column chromatography over silica gel and eluted with,4,6,7,9,10-hexahydro-1,8(2H,5H)acridinedione-10-yl)-thiourea (7a). Yield $74 \%$; brown; mp 130-132 ${ }^{\circ} \mathrm{C}$; IR (KBr) 3420, 3350, 1670, 1445, $1375 \mathrm{~cm}^{-1}$. ${ }^{1} \mathrm{H}$ NMR (90 MHz, $\left.\mathrm{CDCl}_{3}\right) \delta 1.08$ (s, 12H, gem-dimethyl), 2.29 (br s, 8H, $\mathrm{C}_{2}, \mathrm{C}_{4}, \mathrm{C}_{5} \& \mathrm{C}_{7}, \mathrm{CH}_{2}$ ), 3.16 (s, 2H, $\mathrm{C}_{9^{-}}$ $\mathrm{CH}_{2}$ ), 6.6 (s, $\mathrm{NH}_{2}$, exchanged with $\mathrm{D}_{2} \mathrm{O}$ ); $\mathrm{MS}$ (\%) 313 (14.1), 296 (7.2), 272 (18.1), 271 (68.9), 257 (11.7), 256(35.8), 216 (32.8), 214 (100), 91 (22), 83 (27.1), 69 (29.6). $\mathrm{C}_{18} \mathrm{H}_{25} \mathrm{~N}_{3} \mathrm{O}_{2} \mathrm{~S}$ requires: $\mathrm{C}, 62.21 ; \mathrm{H}, 7.25 ; \mathrm{N}, 12.09$. found $\mathrm{C}, 61.93 ; \mathrm{H}, 7.43 ; \mathrm{N}, 11.93$.

$\mathrm{N}$-[9-(4-Hydroxy-3-methoxyphenyl)-3,3,6,6-tetramethyl-3,4,6,7,9,10-hexahydro-1,8(2H, $5 \mathrm{H}$ ) acridinedione -10-yl] thiourea (7b). Yield 81\%; pale brown; mp 212-214 ${ }^{\circ} \mathrm{C}$; IR (KBr) 3450, $3320,1658,1434,1385 \mathrm{~cm}^{-1} .{ }^{1} \mathrm{H}$ NMR $\left(400 \mathrm{MHz}, \mathrm{CDCl}_{3}\right) \delta 0.84 \& 1.01(2 \mathrm{~s}, 12 \mathrm{H}$, gemdimethyl), 1.95-2.17 (2d, 4H,J=16.1, $\left.\mathrm{C}_{2}, \mathrm{C}_{7}-\mathrm{CH}_{2}\right), 2.21-2.42\left(2 \mathrm{~d}, 4 \mathrm{H}, \mathrm{J}=17.33, \mathrm{C}_{4}, \mathrm{C}_{5}-\mathrm{CH}_{2}\right)$, 3.68(s, 3H, $\left.\mathrm{OCH}_{3}\right), 3.71\left(\mathrm{~s}, 2 \mathrm{H}, \mathrm{NH}_{2}\right.$, exchanged with $\left.\mathrm{D}_{2} \mathrm{O}\right), 4.76\left(\mathrm{~s}, 1 \mathrm{H}, \mathrm{C}_{9}-\mathrm{CH}\right), 6.48-7.0(\mathrm{~m}$, $3 \mathrm{H}$, Ar-H), 8.1 \& 8.2 (br s, NH, exchanged with $\mathrm{D}_{2} \mathrm{O}$ ); MS (\%) 393 (35.9), 272 (26.5), 216 (24.9), 91 (23.8), 83 (54.2), 69 (25.7). 


\section{General procedure for the synthesis of acridinedione 8a,b}

A mixture of the tetraketone 1e (1f) $(2.49 \mathrm{mmol})$ and $\mathrm{N}$-aminoethyl piperazine $(2.49 \mathrm{mmol})$ was refluxed in acetic acid $(15 \mathrm{ml})$ for 14 hours. The reaction mixture was cooled and poured into crushed ice. The solid obtained was filtered and purified by column chromatography over silica gel and eluted with $\mathrm{CHCl}_{3}-\mathrm{MeOH}(9: 1)$, to isolate the respective acridinedione $8 \mathrm{a}(8 \mathrm{~b})$.

10-[2-(4-Acetylpiperazin-1-yl)ethyl]-9-(4-methoxyphenyl)-3,3,6,6-tetramethyl-3,4,6, 7, 9,10hexahydro-1,8(2H, 5H) acridinedione (8a). Yield 63\%; pale brown; mp 198-200 ${ }^{\circ} \mathrm{C}$; $\mathrm{IR}(\mathrm{KBr})$ $1685,1665,1547,1374 \mathrm{~cm}^{-1} .{ }^{1} \mathrm{H}$ NMR $\left(400 \mathrm{MHz}, \mathrm{CDCl}_{3}\right): \delta 1.00 \& 1.08(2 \mathrm{~s}, 12 \mathrm{H}$, gemdimethyl), 2.09-3.63 (m, 23H, C $\left.2, \mathrm{C}_{4}, \mathrm{C}_{5}, \mathrm{C}_{7}-\mathrm{CH}_{2}, \mathrm{~N}-\mathrm{CH}_{2}, \mathrm{CH}_{3} \mathrm{CO}\right), 3.71\left(\mathrm{~s}, 3 \mathrm{H}, \mathrm{OCH}_{3}\right), 5.19$ (s, $\left.1 \mathrm{H}, \mathrm{C}_{9} \mathrm{CH}\right), 6.68$ (d, 2H, J=8.7Hz, Ar-H), 7.65 (d, 2H, J=8.3Hz, Ar-H); ${ }^{13} \mathrm{C}$ NMR $(100.4 \mathrm{MHz}$, $\left.\mathrm{CDCl}_{3}\right) \delta 21.2,28.0,29.2,30.8,32.4,40.5,42.1,45.9,49.8,53.1,55.1,113.2,115.7,128.4$, 138.1, 149.8, 157.5, 168.9, 195.6; MS $533\left(\mathrm{M}^{+}, 13.6\right), 491$ (35.9), 395 ( 40.9), 393 (6), 378 (7.3), 362 (4.1), 272 (13.6), 271 (5), 256 (3.4), 83 (13.7), 69 (6.4), 55 (14.4).

10-[2-(4-Acetylpiperazin-1-yl)ethyl]-9-(4-chlorophenyl)-3,3,6,6-tetramethyl-3,4,6, 7,9,10hexahydro-1,8(2H, $\mathbf{5 H}$ ) acridinedione (8b). Yield $75 \%$; brown; mp 212-214 ${ }^{\circ} \mathrm{C}$; IR (KBr) 1680, 1658, 1574, $1380 \mathrm{~cm}^{-1} .{ }^{1} \mathrm{H}$ NMR $\left(200 \mathrm{MHz}, \mathrm{CDCl}_{3}\right): \delta 1.00 \& 1.09(2 \mathrm{~s}, 12 \mathrm{H}$,gemdimethyl), 2.04-2.22 (m, 10H, $\left.\mathrm{CH}_{2}\right), 2.36-2.57\left(\mathrm{~m}, 11 \mathrm{H}, \mathrm{CH}_{2}\right), 5.22(\mathrm{~s}, 1 \mathrm{H}, \mathrm{C} 9-\mathrm{H}), 7.11$ (d, 2H, $=6.3 \mathrm{~Hz}, \mathrm{Ar}-\mathrm{H}), 7.18(\mathrm{~d}, 2 \mathrm{H}, J=6.3 \mathrm{~Hz}, \mathrm{Ar}-\mathrm{H}) ;{ }^{13} \mathrm{C} \mathrm{NMR}\left(50 \mathrm{MHz}, \mathrm{CDCl}_{3}\right): 21.8,28.6,29.9$, 32.0, 33.1, 41.7, 42.8, 46.6, 50.4, 54.7, 115.9, 128.6, 129.6, 144.9, 150.6, 169.6, 196.1.MS 537 $\left(\mathrm{M}^{+}, 48\right), 384$ (21.9), 369 (20.6), 272 (34.2), 270 (40.8), 256 (17.9), 83 (28.9), 69 (16.1), 55 (11.3). $\mathrm{C}_{31} \mathrm{H}_{40} \mathrm{~N}_{3} \mathrm{O}_{3} \mathrm{Cl}$ requires: $\mathrm{C}, 69.19 ; \mathrm{H}, 7.49 ; \mathrm{N}, 7.80$; found: $\mathrm{C}, 68.95 ; \mathrm{H}, 7.28 ; \mathrm{N}, 7.62$.

9- (4- Hydroxy- 3- methoxyphenyl)- 3, 3, 6, 6-tetramethyl- 3, 4, 6, 7, 9, 10-hexa- hydro-1, $\mathbf{8}(2 \mathrm{H}, 5 \mathrm{H})$ acridinedione (9a). General procedure.

A mixture of the tetraketone $1 \mathbf{d}(2.41 \mathrm{mmol})$ and the respective amine $(2.41 \mathrm{mmol})$ was refluxed in acetic acid (15ml) for 5-6 hours. The reaction mixture was cooled and poured into crushed ice. The solid obtained was filtered and purified by column chromatography over silica gel and eluting with $\mathrm{CHCl}_{3}-\mathrm{MeOH}(9: 1)$, to isolate the respective acridinedione 9a-f.

Yield $84 \%$; yellow; mp 296-298 ${ }^{\circ} \mathrm{C}$; IR (KBr) 3273, 3167, 1634, $1499 \mathrm{~cm}^{-1}$; ${ }^{1} \mathrm{H}$ NMR $\left(400 \mathrm{MHz}, \mathrm{CDCl}_{3}-\mathrm{DMSO}-d_{6}\right) \delta 0.95$ and 1.07 (2s, 12H, gem-dimethyl), 2.06-2.21 (2d, 4H, $J=$ $\left.16.3 \mathrm{~Hz}, \mathrm{C}_{4} \& \mathrm{C}_{5}-\mathrm{CH}_{2}\right), 2.30-2.42\left(2 \mathrm{~d}, 4 \mathrm{H}, J=17 \mathrm{~Hz}, \mathrm{C}_{2} \& \mathrm{C}_{7}-\mathrm{CH}_{2}\right.$ ), 3.77(s, 3H, OCH $), 4.85$ (s, 1H, C9-CH), 6.61 (s, 2H, Ar-H), 6.85 (s, 1H, Ar-H), 8.08 (br s, 1H, OH), 8.94 (s, 1H, NH); ${ }^{13} \mathrm{C}$ NMR (100.4 MHz, CDCl $\left.3-\mathrm{DMSO}-d_{6}\right) \delta 25.6\left(\mathrm{CH}_{3}\right), 28.3\left(\mathrm{CH}_{3}\right), 31.1(\mathrm{C}), 31.3(\mathrm{CH}), 49.5$ $\left(\mathrm{CH}_{2}\right), 54.4\left(\mathrm{OCH}_{3}\right), 110.9(\mathrm{CH}), 111.4(\mathrm{C}), 113.5(\mathrm{CH}), 118.8(\mathrm{CH}), 137.6(\mathrm{C}), 142.9(\mathrm{C}), 145.4$ (C), 147.8(C), 194.0 (CO). MS 395 (93.3, M ), 394 (16.9), 380 (10.9), 379 (4.1), 378(9), 350 (3.9), 273 (46), 272 (100), 271 (14.3), 256 (9.3), 228 (4.4), 217 (3.2), 189 (3.6), 124 (8.9), 109 (5.8), 81 (4.5), 53 (3); $\mathrm{C}_{24} \mathrm{H}_{29} \mathrm{NO}_{4}$ requires: $\mathrm{C}, 72.88 ; \mathrm{H}, 7.39 ; \mathrm{N}, 3.54$; found: $\mathrm{C}, 73.11 ; \mathrm{H}, 7.53$; N, 3.26.

9- (4-Hydroxy-3-methoxyphenyl)-10-carboxymethyl-3, 3, 6, 6- tetramethyl-3, 4, 6, 7,9, 10 hexahydro-1, 8(2H, 5H) acridinedione (9b). Yield $64 \%$; yellow; mp 237-239 C; IR (KBr) 
3150, 2982, 1720, $1640,1371 \mathrm{~cm}^{-1} ;{ }^{1} \mathrm{H}$ NMR (400 MHz, CDCl 3 -DMSO- $\left.d_{6}\right) \delta 0.97$ and 1.07 (2s, $12 \mathrm{H}$, gem-dimethyl), 2.12-2.22 (2d, $\left.4 \mathrm{H}, J=16 \mathrm{~Hz}, \mathrm{C}_{4} \& \mathrm{C}_{5}-\mathrm{CH}_{2}\right), 2.35-2.49(2 \mathrm{~d}, 4 \mathrm{H}, J=$ $\left.17 \mathrm{~Hz}, \mathrm{C}_{2} \& \mathrm{C}_{7}-\mathrm{CH}_{2}\right), 3.35$ (s, $\left.2 \mathrm{H}, \mathrm{N}-\mathrm{CH}_{2}\right), 3.79$ (s, 3H, $\left.\mathrm{OCH}_{3}\right), 5.05$ (s, $\left.1 \mathrm{H}, \mathrm{C}_{9}-\mathrm{CH}\right), 6.61-6.93$ (m, 3H, Ar-H); MS 380 (1, M+73), 124 (85), 109 (100), 81 (45), 53 (15). The X-ray diffraction studies established the structure of $9 b^{25}$

9- (4- Hydroxy- 3- methoxyphenyl)-10- (4- methylphenyl)- 3, 3, 6, 6- tetramethyl-3, 4, 6, 7,9, 10- hexahydro-1, 8(2H, $\mathbf{5 H}$ ) acridinedione (9c). Yield $77 \%$; dark brown; mp 176-178 ${ }^{\circ} \mathrm{C}$; IR (KBr) 3404, 2956, 1635, $1364 \mathrm{~cm}^{-1} ;{ }^{1} \mathrm{H}$ NMR $\left(400 \mathrm{MHz}, \mathrm{CDCl}_{3}\right.$-DMSO-d $\left.d_{6}\right) \delta 0.81$ and $0.94(2 \mathrm{~s}$, 12H, gem-dimethyl), 1.81-2.10 (2d, 4H, J=17.58 Hz, $\left.\mathrm{C}_{4} \& \mathrm{C}_{5}-\mathrm{CH}_{2}\right), 2.10-2.21(2 \mathrm{~d}, 4 \mathrm{H}, J=16.6$ $\mathrm{Hz}, \mathrm{C}_{2} \& \mathrm{C}_{7}-\mathrm{CH}_{2}$ ), 2.47 (s, 3H, Ar- $\left.\mathrm{CH}_{3}\right), 3.88$ (s, 3H, OCH $), 5.16$ (s, $\left.1 \mathrm{H}, \mathrm{C}_{9}-\mathrm{CH}\right), 6.50(\mathrm{~s}, 1 \mathrm{H}$, $\mathrm{OH}), 6.75-7.36(\mathrm{~m}, 7 \mathrm{H}, \mathrm{Ar}-\mathrm{H}) ;{ }^{13} \mathrm{C} \mathrm{NMR}\left(100.4 \mathrm{MHz}, \mathrm{CDCl}_{3}-\mathrm{DMSO}-d_{6}\right) \delta 20.9\left(\mathrm{Ar}^{-\mathrm{CH}_{3}}\right), 26.3$ $\left(\mathrm{CH}_{3}\right), 29.4\left(\mathrm{CH}_{3}\right),(\mathrm{CH}), 32.0(\mathrm{C}), 41.4\left(\mathrm{CH}_{2}\right), 49.9\left(\mathrm{CH}_{2}\right), 55.5\left(\mathrm{OCH}_{3}\right), 111.5(\mathrm{CH}), 114.0$ $(\mathrm{CH}), 114.2(\mathrm{C}), 119.1(\mathrm{CH}),(129.3(\mathrm{CH})$ and $130.4(\mathrm{CH})$ signals were distorted),

135.9 (C), 138.1 (C), 139.1 (C), 143.6 (C), 146.0 (C), 149.6 (C), 195.7 (CO); MS 485 (56.8, $\mathrm{M}^{+}$), 362 (66.2). $\mathrm{C}_{31} \mathrm{H}_{35} \mathrm{NO}_{4}$ requires: $\mathrm{C}, 76.67 ; \mathrm{H}, 7.26$; N, 2.88; found: $\mathrm{C}, 76.77 ; \mathrm{H}, 7.54 ; \mathrm{N}, 2.29$.

9- (4- Hydroxy- 3- methoxyphenyl)- 10-(4-methoxyphenyl)- 3, 3, 6, 6- tetramethyl- 3, 4, 6, 7,9, 10- hexahydro-1, 8(2H, 5H) acridinedione (9d). Yield $79 \%$; brown; mp 253-255 ${ }^{\circ} \mathrm{C}$; IR (KBr) 3390, 2967, 1660, $1342 \mathrm{~cm}^{-1} ;{ }^{1} \mathrm{H}$ NMR (400 MHz, CDCl $\left.3-\mathrm{DMSO}_{3} \mathrm{~d}_{6}\right) \delta 0.82$ and $0.95(2 \mathrm{~s}$, 12H, gem-dimethyl), 1.83-2.09 (2d, 4H, J $\left.=17.6 \mathrm{~Hz}, \mathrm{C}_{4} \& \mathrm{C}_{5}-\mathrm{CH}_{2}\right), 2.12-2.22(2 \mathrm{~d}, 4 \mathrm{H}, \mathrm{J}=$ $\left.16.3 \mathrm{~Hz}, \mathrm{C}_{2} \& \mathrm{C}_{7}-\mathrm{CH}_{2}\right), 3.90 \& 3.91\left(2 \mathrm{~s}, 6 \mathrm{H}, \mathrm{Ar}-\mathrm{OCH}_{3}\right), 5.18\left(\mathrm{~s}, 1 \mathrm{H}, \mathrm{C}_{9}-\mathrm{CH}\right), 5.57(\mathrm{~s}, 1 \mathrm{H}, \mathrm{Ar}-$ $\mathrm{OH}), 6.73-7.13(\mathrm{~m}, 7 \mathrm{H}, \mathrm{Ar}-\mathrm{H}) ;{ }^{13} \mathrm{C}$ NMR $\left(100.4 \mathrm{MHz}, \mathrm{CDCl}_{3}\right.$-DMSO-d $\left._{6}\right) \delta 26.7\left(\mathrm{CH}_{3}\right), 29.7$ $\left(\mathrm{CH}_{3}\right), 32.0(\mathrm{CH}), 32.2(\mathrm{C}), 41.7\left(\mathrm{CH}_{2}\right), 50.1\left(\mathrm{CH}_{2}\right), 55.5\left(\mathrm{OCH}_{3}\right), 55.7\left(\mathrm{OCH}_{3}\right), 111.8(\mathrm{CH})$, $113.8 \mathrm{CH}), 114.6(\mathrm{C}), 119.2(\mathrm{CH}), 129.9(\mathrm{CH}), 130.8(\mathrm{CH}), 131.4(\mathrm{C}), 138.6(\mathrm{C}), 143.6(\mathrm{C})$, 145.8 (C), 150.0 (C), 159.7 (C), 195.9 (CO); MS $501\left(100, \mathrm{M}^{+}\right), 500$ (14), 486 (12.3), 485 (11.3), 473 (7.2), 416 (7.7), 379 (79), 378 (100), 363 (8.2), 362 (19.5), 348 (7.7), 334 (7.9), 322 (10.5), 320 (5.7), 250.5 (31.6). $\mathrm{C}_{31} \mathrm{H}_{35} \mathrm{NO}_{5}$ requires: $\mathrm{C}, 74.22 ; \mathrm{H}, 7.03$; N, 2.79; found $\mathrm{C}$, 73.91; $\mathrm{H}, 6.99$; $\mathrm{N}, 2.48$.

9- (4- Hydroxy- 3- methoxyphenyl)- 10- phenyl- 3, 3, 6, 6- tetramethyl- 3, 4, 6, 7,9, 10hexahydro-1, 8(2H, 5H) acridinedione (9e). Yield $75 \%$; brown; mp 232-234 $\mathrm{C}$; $\mathrm{IR}(\mathrm{KBr}) 3410,2967,1645,1350 \mathrm{~cm}^{-1} ;{ }^{1} \mathrm{H}$ NMR $\left(400 \mathrm{MHz}, \mathrm{CDCl}_{3}\right.$-DMSO-d $\left.\mathrm{d}_{6}\right) \delta 0.81$ and 0.94 (2s, $12 \mathrm{H}$, gem-dimethyl), 1.79-2.09 (2d, $\left.4 \mathrm{H}, J=17.3 \mathrm{~Hz}, \mathrm{C}_{4} \& \mathrm{C}_{5}-\mathrm{CH}_{2}\right), 2.12-2.22(2 \mathrm{~d}, 4 \mathrm{H}$, $\left.J=16.8 \mathrm{~Hz}, \mathrm{C}_{2} \& \mathrm{C}_{7}-\mathrm{CH}_{2}\right), 3.89$ (s, $\left.3 \mathrm{H}, \mathrm{OCH}_{3}\right), 5.20\left(\mathrm{~s}, 1 \mathrm{H}, \mathrm{C}_{9}-\mathrm{CH}\right), 5.65$ (s, $\left.1 \mathrm{H}, \mathrm{Ar}-\mathrm{OH}\right), 6.77-$ $7.56(\mathrm{~m}, 8 \mathrm{H}, \mathrm{Ar}-\mathrm{H}) ;{ }^{13} \mathrm{C} \mathrm{NMR}\left(100.4 \mathrm{MHz}, \mathrm{CDCl}_{3}-\mathrm{DMSO}-\mathrm{d}_{6}\right) \delta 26.6\left(\mathrm{CH}_{3}\right), 29.6\left(\mathrm{CH}_{3}\right), 31.9$ $(\mathrm{CH}), 32.3(\mathrm{C}), 41.7\left(\mathrm{CH}_{2}\right), 50.1\left(\mathrm{CH}_{2}\right), 55.8\left(\mathrm{OCH}_{3}\right), 111.8(\mathrm{CH}), 113.9(\mathrm{CH}), 114.7(\mathrm{C}), 119.2$ $(\mathrm{CH}),(129.3(\mathrm{CH})$, and 130.1 (CH) signals were distorted) $138.5(\mathrm{C}), 138.9(\mathrm{C}), 143.6(\mathrm{C}), 145.8$ (C), 149.5 (C), 196.0 (CO); MS471 (69.9, M+), 456 (5.4), 454 (4.3), 348 (100), 333 (3.9), 332 (13.5). $\mathrm{C}_{30} \mathrm{H}_{33} \mathrm{NO}_{4}$ requires: $\mathrm{C}, 76.40 ; \mathrm{H}, 7.05 ; \mathrm{N}, 2.94$; found $\mathrm{C}, 76.21 ; \mathrm{H}, 6.99 ; \mathrm{N}, 2.78$.

9- (4- Hydroxy- 3- methoxyphenyl)- 10- (3,4-dimethylphenyl)- 3, 3, 6, 6-tetramethyl-3, 4, 6, 7,9, 10- hexahydro- 1, 8(2H, 5H) acridinedione (9f). Yield 76\%; brown; mp 260-262 ${ }^{\circ} \mathrm{C}$; $\operatorname{IR}(\mathrm{KBr}) 3403,2955,1634,1364 \mathrm{~cm}^{-1} ;{ }^{1} \mathrm{H}$ NMR (400 MHz, $\left.\mathrm{CDCl}_{3}-\mathrm{DMSO}_{-} \mathrm{d}_{6}\right) \delta 0.82$ and 0.96 
(2s, 12H, gem-dimethyl), 1.82-2.14 (2d, 4H, J=17.6 Hz, $\left.\mathrm{C}_{4} \& \mathrm{C}_{5}-\mathrm{CH}_{2}\right), 2.10-2.20(2 \mathrm{~d}, 4 \mathrm{H}$, $\left.J=16.12 \mathrm{~Hz}, \mathrm{C}_{2} \& \mathrm{C}_{7}-\mathbf{C H}_{2}\right), 2.36\left(\mathrm{~s}, 3 \mathrm{H}, \mathrm{Ar}-\mathrm{CH}_{3}\right), 2.37\left(\mathrm{~s}, 3 \mathrm{H}, \mathrm{Ar}-\mathrm{CH}_{3}\right), 3.84\left(\mathrm{~s}, 3 \mathrm{H}, \mathrm{OCH}_{3}\right)$, 5.08 (s, 1H, 9-CH), 6.71-8.03 (m, 7H, Ar-H and OH); $\left.{ }^{13} \mathrm{C} \mathrm{NMR} \mathrm{(100.4} \mathrm{MHz,} \mathrm{CDCl}_{3}-\mathrm{DMSO}-d_{6}\right)$

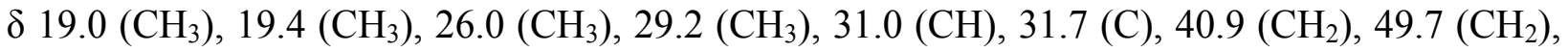
$55.1\left(\mathrm{OCH}_{3}\right), 111.2(\mathrm{CH}), 113.6(\mathrm{C}), 114.4(\mathrm{CH}), 119.1(\mathrm{CH}),(127,129$ and 130 were distorted signals) 135.7 (C), 137.5 (C), 143.8 (C), 146.3 (C), 149.7 (C), 195.2 (CO); MS 499 (75.4, M ), $376(100) . \mathrm{C}_{32} \mathrm{H}_{37} \mathrm{NO}_{2}$ requires: $\mathrm{C}, 76.92 ; \mathrm{H}, 7.46 ; \mathrm{N}, 2.80$; found $\mathrm{C}, 76.56 ; \mathrm{H}, 7.31 ; \mathrm{N}, 2.63$.

10 - (4 - Hydroxyphenyl) - 3, 4, 6, 7, 9, 10-hexahydro - 1, 8 (2H, 5H) acridinedione (10a). General procedure to give the acridinedio. The tetraketone 1a $(1.0 \mathrm{~g}, 4.2 \mathrm{mmol})$ and $p$ aminophenol $(0.46 \mathrm{~g}, 4.2 \mathrm{mmol})$ were refluxed in acetic acid for 12 hours. The reaction mixture was cooled, filtered and dried ne 10a.

Yield $69 \%$; brown; mp 255-257 $\mathrm{C}$; IR (KBr) 3280, 1655, 1536, $1373 \mathrm{~cm}^{-1} ;{ }^{1} \mathrm{H}$ NMR (300 MHz, $\left.\mathrm{CDCl}_{3}-\mathrm{DMSO}-d_{6}\right) \delta$ 1.6-2.2 (m, 12H, $\left.\mathrm{C}_{2}, \mathrm{C}_{3}, \mathrm{C}_{4}, \mathrm{C}_{5}, \mathrm{C}_{6} \& \mathrm{C}_{7}-\mathrm{CH}_{2}\right), 2.93\left(\mathrm{~s}, 2 \mathrm{H}, \mathrm{C}_{9}-\mathrm{CH}_{2}\right), 6.82$ $(\mathrm{d}, 2 \mathrm{H}, J=8.8 \mathrm{~Hz}, \mathrm{Ar}-\mathrm{H}), 7.11(\mathrm{~d}, 2 \mathrm{H}, J=8.8 \mathrm{~Hz}, \mathrm{Ar}-\mathrm{H}), 9.89$ (s, 1H, Ar-OH); ${ }^{13} \mathrm{C}$ NMR $\left(300 \mathrm{MHz}, \mathrm{CDCl}_{3}-\mathrm{DMSO}_{6}\right.$ ) $\delta$ 19.3, 21.6, 28.2, 36.5,110.6, 116.6, 130.5, 131.4, 154.6, 158.2, 196.6; MS $309\left(\mathrm{M}^{+}, 1\right) . \mathrm{C}_{19} \mathrm{H}_{19} \mathrm{NO}_{3}$ requires: $\mathrm{C}$, 73.76; $\mathrm{H}, 6.18$; $\mathrm{N}, 4.52$; found $\mathrm{C}, 73.40 ; \mathrm{H}, 6.26$; $\mathrm{N}, 4.25$.

10-(4-Hydroxyphenyl)- 3,3,6,6-tetramethyl-3, 4,6,7,9,10-hexahydro-1,8(2H,5H) acridinedione (10b). Yield $76 \%$; pale green; mp above $300^{\circ} \mathrm{C}$; IR (KBr) 3180, 1640, 1555, $1390 \mathrm{~cm}^{-1} ;{ }^{1} \mathrm{H}$ NMR (300 MHz, CDCl 3 -DMSO-d 6 ) $\delta 0.88\left(\mathrm{~s}, 12 \mathrm{H}\right.$, gem-dimethyl), $2.12\left(\mathrm{~s}, 4 \mathrm{H}, \mathrm{C}_{2} \& \mathrm{C}_{7}-\mathrm{CH}_{2}\right)$, $2.25\left(\mathrm{~s}, 4 \mathrm{H}, \mathrm{C}_{4} \& \mathrm{C}_{5}-\mathrm{CH}_{2}\right), 2.98\left(\mathrm{~s}, 2 \mathrm{H}, \mathrm{C}_{9}-\mathrm{CH}_{2}\right), 6.85$ (d, 2H, J = 8.4 Hz, Ar-H), 7.06 (d, 2H, $=7.5 \mathrm{~Hz}, \mathrm{Ar}-\mathrm{H}), 9.87(\mathrm{~s}, 1 \mathrm{H}, \mathrm{Ar}-\mathrm{OH}) ;{ }^{13} \mathrm{C} \mathrm{NMR}\left(300 \mathrm{MHz}, \mathrm{CDCl}_{3}-\mathrm{DMSO}-d_{6}\right) \delta 18.5,27.7,31.8$, 45.4, 49.4, 108.7, 109.0, 116, 130.6, 151.8, 195.5; MS $365\left(\mathrm{M}^{+}, 9\right), 348$ (31), 337 (1), 323 (2), 309 (1), 273 (100), 272 (8). $\mathrm{C}_{23} \mathrm{H}_{27} \mathrm{NO}_{3}$ requires: $\mathrm{C}, 75.59$; $\mathrm{H}, 7.45 ; \mathrm{N}, 3.83$; found $\mathrm{C}, 75.38$; $\mathrm{H}$, $7.57 ; \mathrm{N}, 3.76$.

10 - (4-Hydroxyphenethyl) 3,3,6,6-tetramethyl-- 3, 4, 6, 7, 9, 10 - hexahydro -1, 8 (2H, 5H) acridinedione (10c). Yield $85 \%$; brown; $\mathrm{mp} 236-238^{\circ} \mathrm{C}$; IR (KBr) 3197, 1643, 1561, 1395 $\mathrm{cm}^{-1}$; ${ }^{1} \mathrm{H}$ NMR (400 MHz, CDCl 3 -DMSO-d $\left.)_{6}\right) \delta 1.03$ (s, 12H, gem-dimethyl), 2.18 (s, 4H, C 2 , \& $\mathrm{C}_{4^{-}}$ $\left.\mathrm{CH}_{2}\right), 2.36\left(\mathrm{~s}, 4 \mathrm{H}, \mathrm{C}_{5} \& \mathrm{C}_{7}-\mathrm{CH}_{2}\right), 2.73\left(\mathrm{t}, 2 \mathrm{H}, \mathrm{J}=6.5 \mathrm{~Hz}, \mathrm{Ar}-\mathrm{CH}_{2}\right), 3.05\left(\mathrm{~s}, 2 \mathrm{H}, \mathrm{C}_{9}-\mathrm{CH}_{2}\right), 3.76(\mathrm{t}$, $\left.2 \mathrm{H}, J=6.5 \mathrm{~Hz}, \mathrm{~N}-\mathrm{CH}_{2}\right), 6.79$ (d, 2H, $\left.J=8.3 \mathrm{~Hz}, \mathrm{Ar}-\mathrm{H}\right), 6.99$ (d, 2H, $\left.J=8.3 \mathrm{~Hz}, \mathrm{Ar}-\mathrm{H}\right), 8.72$ (br s, $1 \mathrm{H}, \mathrm{Ar}-\mathrm{OH}) ;{ }^{13} \mathrm{C} \mathrm{NMR}\left(100.4 \mathrm{MHz}, \mathrm{CDCl}_{3}-\mathrm{DMSO}-d_{6}\right) \delta 17.5\left(\mathrm{CH}_{2}\right), 28.2\left(\mathrm{CH}_{3}\right), 31.9(\mathrm{C})$, $36.3\left(\mathrm{CH}_{2}\right), 40.1\left(\mathrm{CH}_{2}\right), 45.9\left(\mathrm{CH}_{2}\right), 49.3\left(\mathrm{CH}_{2}\right), 111.6(\mathrm{C}), 115.5(\mathrm{CH}), 127.3(\mathrm{C}), 129.6(\mathrm{CH})$, 151.6 (C), 156.2 (C), 196.2 (CO); MS 393 (M+, 39), 300 (41), 272 (58),131 (16), 120 (60), 117 (44), 94 (27), 91(48), 84 (81). $\mathrm{C}_{25} \mathrm{H}_{31} \mathrm{NO}_{3}$ requires: $\mathrm{C}, 76.30 ; \mathrm{H}, 7.93$; N, 3.56; found $\mathrm{C}, 76.39$; $\mathrm{H}, 8.01 ; \mathrm{N}, 3.23$.

10 - (4-Hydroxyphenethyl)-9-methyl-3, 4, 6, 7, 9, 10-hexahydro -1, $8(2 \mathrm{H}, 5 \mathrm{H})$ acridinedione (10d). Yield $71 \%$; yellow; mp 271-273 ${ }^{\circ} \mathrm{C}$; IR (KBr) 3210, 1657, 1580, 1398 $\mathrm{cm}^{-1}$; ${ }^{1} \mathrm{H}$ NMR $\left(400 \mathrm{MHz}, \mathrm{CDCl}_{3}-\mathrm{DMSO}-d_{6}\right) \delta 0.82\left(\mathrm{~d}, 3 \mathrm{H}, J=6.8 \mathrm{~Hz}, \mathrm{CH}_{3}\right), 1.90-2.64\left(\mathrm{~m}, 12 \mathrm{H}, \mathrm{C}_{2}, \mathrm{C}_{3}, \mathrm{C}_{4}, \mathrm{C}_{5}\right.$, $\left.\mathrm{C}_{6} \& \mathrm{C}_{7}-\mathrm{CH}_{2}\right), 2.77\left(\mathrm{t}, 2 \mathrm{H}, J=7.3 \mathrm{~Hz}, \mathrm{Ar}-\mathrm{CH}_{2}\right), 3.82\left(\mathrm{t}, 2 \mathrm{H}, J=7.3 \mathrm{~Hz}, \mathrm{~N}-\mathrm{CH}_{2}\right), 3.95(\mathrm{q}, 1 \mathrm{H}$, $\mathrm{CH}_{3}$ ), $6.76(\mathrm{~d}, 2 \mathrm{H}, J=8.3 \mathrm{~Hz}, \mathrm{Ar}-\mathrm{H}), 7.02$ (d, 2H, $\left.J=8.3 \mathrm{~Hz}, \mathrm{Ar}-\mathrm{H}\right), 9.03$ (br s, 1H, Ar-OH); ${ }^{13} \mathrm{C}$ NMR (100.4 MHz, $\left.\mathrm{CDCl}_{3}-\mathrm{DMSO}-d_{6}\right) \delta 120.0,20.4,21.0,25.5,29.9,35.4,45.4,114.6$, 116.3, 126.5, 128.7, 151.4, 155.3, 195.0. MS 336 (100), 216 (21). $\mathrm{C}_{22} \mathrm{H}_{25} \mathrm{NO}_{3}$ requires: $\mathrm{C}, 75.18$; H, 7.17; N, 3.98; found C, 74.98; H, 7.18; N, 3.64 . 


\section{Acknowledgments}

The authors thank CSIR, New Delhi, for financial assistance and University Grants Commission, New Delhi, for Special Assistance Programme to the Department of Organic Chemistry.

\section{References}

1. Beer, D. P. Chem. Comm. 1966, 689.

2. Lee, D. H.; Lee, K. H.; Hong, J. Org. Lett. 2001, 3, 5.

3. Czarnik, A. W. ACS Symposium Series 1992, 538, 25.

4. Nishizawa, S.; Kato, R.; Hayashita, T.; Teramae, N. Anal.Sci. 1988, 14, 595.

5. Gunnlaugsson, T.; Davis, A. P.; Glynn, M. Chem. Commun. 2001, 2556.

6. Janis, R. A.; Silver, P. J.; Triggle, D. J. Adv. Drug Res. 1987, 16, 309.

7. Bossert, F. ; Meyer, H.; Wehinger, E. Angew. Chem., Int. Ed. 1981, 20, 762.

8. Bossert, F.; Vater, W. Naturwis 1971, 58, 578.

9. Bossert, F.; Vater, W. Med. Res. Rev. 1989, 9, 291.

10. Martin, N.; Seoane, C. Quim. Ind. 1990, 36, 115.

11. Jani, R. A.; Triggle, D. J. J. Med. Chem. 1983, 26, 775.

12. Goldmann, S.; Stoltefuss, J. Angew. Chem., Int. Ed. 1991, 30, 1559.

13. Hofmann, H.; Camiraglia, R. J. J. Mol. Struct. TEOCHEM 1990, 205, 1.

14. Meyer, H.; Bossert, F.; Horstmann Liebigs Ann. Chem. 1976, 1762.

15. Love, B.; Goodmann, M.; Snader, K.; Tedeschi, R.; Macko, E. J. Med. Chem. 1974, 17, 956.

16. Schramm, M.; Thomas, G.; Tower, R. Nature 1983, 303, 535.

17. Shanmugasundaram, P.; Prabahar, K. J.; Ramakrishnan, V. T. J. Heterocyclic Chem. 1993, 30, 1003.

18. Murugan, P.; Shanmugasundram, P.; Ramakrishnan, V. T.; Venkatachalapathy, B.; Srividya, N.; Ramamurthy, P.; Gunasekaran, K.; Velmurugan, D. J. Chem. Soc., Perkin Trans. 2 1998, 2, 999.

19. Shanmugasundaram, P.; Prabahar, K. J.; Ramakrishnan, V. T.; Srividya, N.; Ramamurthy, P. Heteroatom Chem. 1996, 7, 17.

20. Horning, E. C.; Horning, M. G. J. Org. Chem. 1946, 11, 95.

21. Boyd, E. F.; Waldor, M. K. Infection and Immunity 1999, 5898.

22. Acheson, R. M. Acridines; Interscience Publishers, Inc.: New York, 1956.

23. Denny, W.R. The Chemistry of Antitumour agents; Wilman, D.E.V, Ed.; Blackie \& Sons, Ltd: Glasgow and London, 1990.

24. Adcock, B. Acridines: In HeterocyclicCompounds, Weissberger Series, 1973, p 9.

25. Thinagar, S.; Velmurugan, D. Department of Crystallography and Biophysics, University of Madras (Unpublished results). 